RUNNING HEAD: Instructed repetition

Truth by Repetition ... without repetition:

Testing the effect of instructed repetition on truth judgments

\author{
Simone Mattavelli \\ University of Milano-Bicocca \\ Olivier Corneille \\ UCLouvain \\ Christian Unkelbach \\ University of Cologne
}

Corresponding author: Simone Mattavelli, University of Milan-Bicocca, Department of Psychology, 1, Piazza dell'Ateneo Nuovo, 20126, Milan, Italy.

E-mail: simone.mattavelli@unimib.it 


\begin{abstract}
Past research indicates that people judge repeated statements as more true than new ones. An experiential consequence of repetition that may underly this "truth effect" is processing fluency: processing statements feels easier following their repetition. Here, we examine the effect of merely instructed (i.e., not experienced) repetition on truth judgments, which we compared to the effect of factual repetition. In two preregistered experiments $(\mathrm{N}=468)$, we found a larger truth effect for factual repetition compared to instructed repetition. However, we also found a non-experiential contribution to truth judgments: statements instructed to be repeated were judged more true than those instructed to be new. Experiment 2 further clarified that adding a repetition status tag in the factual repetition condition did not impact truth judgments. Moreover, for both experienced and instructed repetition, the effect on truth was qualified by subjective recognition. We discuss the mechanisms that can explain the impact of instructed repetition on truth and its implications for a better understanding of the truth effect.
\end{abstract}

Keywords: truth-by-repetition, instructions, fluency, symbolic repetition 


\section{Truth by Repetition ... without repetition: \\ Testing the effect of instructed repetition on truth judgments}

In a world where information of any kind is available from multiple sources, people often face the task of judging whether information is true or not. One factor influencing their judgments is prior exposure: People judge statements to be more true after being exposed to them. This phenomenon is known as "illusory truth effect", "truth-by-repetition effect", or simply the "truth effect". Initially introduced by Hasher et al. (1977), the truth effect is a robust phenomenon (see Dechêne et al., 2010), at play in many different domains, including consumer advertising (Johar \& Roggeveen, 2007), social-political opinions (Arkes et al., 1989) and rumors (DiFonzo et al., 2016). More recently, the phenomenon has been considered a prominent candidate to explain misinformation effects and even "fake news" (Pennycook et al., 2018). Thus, addressing the mechanisms underlying the truth effect and isolating the conditions under which repetition influences truth is a prominent research goal.

There are several explanations of the truth effect. Bacon (1979) argued that recognizing repeated statements (i.e., judging them as "repeated") is the critical process. Later, Arkes and colleagues (1989) argued that subjective familiarity with the statements increases truth ratings. A construct that contributes to recognition and familiarity is processing fluency: the experienced ease of ongoing mental operations (Clore, 1992; Unkelbach \& Greifeneder, 2013). Reber and Schwarz (1999) showed that manipulating processing by color contrast and not by repetition has similar effects on judged truth. Unkelbach (2007) then established the functional equivalence of fluency due to repetition and fluency due to color contrast. However, "pure" fluency effects are typically smaller than truth-by-repetition effects; thus, Unkelbach and Rom (2017) argued that a referential network formation underlies the truth effect. This referential process also influences the related constructs of recognition, familiarity, and fluency, jointly leading to strong truth-by-repetition effects (see Unkelbach et al., 2019, for a summary). 
Common to all these explanations is the fact that they build on experiences within the mental system, such as fluency and familiarity. ${ }^{1}$ These experiences, and also the formation of a referential network, follow from factual prior exposure. In other words, the factual repetition of the information is the primary determinant for these processing effects to emerge. However, recent and classic theorizing has neglected the non-experiential aspect of the truth effect. Repetition is not only important factually (i.e., as an experience) but also symbolically (i.e., as knowledge). For example, if a newsblog opens with a statement that "Many reports state fact X", readers do not need to look up these other reports to understand that fact $\mathrm{X}$ is repeated. The stated repetition of fact $\mathrm{X}$ communicates information; in the case of a newsblog, most likely, that fact $\mathrm{X}$ is currently of interest to many writers and readers. Similarly, summarizing a group discussion with "Everybody stated fact X" conveys agreement about fact X. Likewise, without repetition actually taking place, stating that fact X occurs repeatedly may communicate information about the truth of fact X. For example, when scrolling down the wall of a social network, one may see a statement appearing ten times, posted by as many users. Alternatively, one may see that very same statement appearing just once and realize from the repost button that ten users have posted it. In the latter case, the user does not directly experience the actual repetition of the target statement. However, merely knowing about the frequency of its occurrence might suffice to affect the statement's truth value. Directly relevant to the present research, people may believe that repeated statements have a higher probability to be factually true (Unkelbach \& Greifeneder, 2013), and thus symbolic repetition may be used as a cue to infer truth even when repetition is not experienced.

Following this rationale, one may ask whether "repetition" factually needs to be realized by prior exposure or if merely informing people that information is repeated influences subjective truth. In other words, one might locate a cause of the truth effect not within the cognitive functioning, such as experiences of recognition, familiarity, or fluency, but in meta-cognitive

\footnotetext{
${ }^{1}$ Familiarity is often conceptualized as the interpretation of processing fluency due to prior occurrence (e.g., Whittlesea, 1990). As both were used as explanations, we treat them as separate, but closely related constructs
} 
functioning (Nelson, 1996). Under this view, the organism imposes meaning on top of the property (i.e., repetition) that induces cognitive experiences. Potentially, the symbolic denotative meaning of repetition rather than the factual connotative experience of repetition leads to a truth effect. Of course, it may also be the case that both experiential and non-experiential processes contribute to the truth effect; if so, it is worth examining to what extent they do.

Prior studies already provide some evidence that the effect of repetition goes beyond the resulting experience. First, Bacon (1979) showed that the subjective recognition of a statement, rather than its actual repetition, predicted its truth value. The authors (i.e., "Bacon" was a pen name for a group of researchers) found that statements presented in a test phase and believed to be repeated (i.e., seen in an initial exposure phase) were judged as more true than those believed to be new, even when the statements had not been factually presented in the exposure phase. If the effect of repetition has more to do with the subjective repetition judgment than with its objective prior exposure, then it becomes plausible that processes other than those initiated by actual repetition might contribute to the truth effect. In other words, simply encoding a statement as "repeated" might suffice to increase its truth. In a second experiment, Bacon also found that repetition increased truth when participants were verbally informed about statements' repetition status: repeated statements became more subjectively true than new ones when participants were told that they were indeed repeated. Again, this suggests that the effect of factual repetition on truth judgments does not dissociate from that of instructed repetition and that a common component might underly both the effects.

Further support for a non-experiential component of the truth effect comes from recent research on the mere-exposure effect. Mere exposure is the enhanced liking for repeated stimuli (Zajonc, 1968, see Bornstein, 1989, and Montoya et al., 2017 for meta-analyses). Similar to the truth effect, processing fluency serves as an explanation for increased liking due to stimulus repetition (Winkielman et al., 2003). Recently, however, Van Dessel et al. (2017) showed that mere exposure effects can also be obtained via verbal information about stimulus repetition. These 
authors provided simple instructions about the repetition status of two alternative non-words (e.g., "The word LOKANTA will be presented often and the word FEVKANI will be presented rarely"). The authors found that non-words instructed to be presented were liked more. In discussing the potential explanations of instruction-based mere-exposure effects, the authors claimed that by acquiring knowledge about the repetition of a stimulus, people may elaborate on this information and infer that more frequent stimuli are positive.

Building on these prior findings, we investigate the role of repetition on truth judgments without factual repetition (i.e., without prior exposure). That is, we tested if repetition increases truth judgments even when participants are solely informed about the repetition status of a statement. Such an instruction-based effect may also point to the role of demand characteristics in the truth effect. When "experiential" effects are replicated on a mere verbal basis, one cannot exclude that the participants in the experienced condition relied on expectations to produce desired effect on a purely inferential - not experiential - basis (Orne, 1962; for a recent discussion, see Corneille \& Lush, 2021).

Experimentally separating experiential and symbolic contributors to TBR effects is no trivial task. In a standard truth-by-repetition paradigm, participants are first exposed to a list of statements, and then, in a subsequent judgment phase, they are asked to estimate the truth value of both repeated and new statements (see Dechêne et al., 2010 for a quantitative review). Under such conditions, repetition and experience do not vary independently, and acquiring knowledge about the repetition of a statement (i.e., repeated vs. new) is conditional upon the subjective experience made with that repetition. Begg and colleagues (1992) used a process dissociation procedure to address this necessary confound and to separate the experiential contribution from the symbolic contribution; knowledge about statements' truth was manipulated by presenting statements paired with either credible (true) or not credible (false) sources. Thus, repeated true statements could be judged true based on both source recollection and the experience (e.g., familiarity, fluency), whereas judging repeated false statements could depend only on source recollection. Using a 
similar method (i.e., multinomial processing trees), Unkelbach and Stahl (2009) also isolated the contribution of experiences to the truth effect.

However, the symbolic contribution of repetition cannot be isolated in a factual repetition paradigm. A statement is only known to be repeated if it is factually repeated, which contaminates the symbolic with the experiential contributions; or, people falsely believe it to be repeated (see above), which also contaminates the repetition belief with the processing of the statements (e.g., a statement feels familiar and is thus judged to be repeated and true). To overcome this limitation, we employed a variant of the paradigm by Van Dessel et al. (2017) to investigate whether instructed repetition influences truth judgments in the absence of prior exposure.

\section{The present research}

Instruction-based procedures experimentally unconfound repetition knowledge from repetition experience; that is, participants are instructed about the assumed state of the world (i.e., repetition) without factually experiencing the state of the world. Thus, the impact of repetition on truth is due to the symbolic meaning of repetition, not the experience or the meaning of the experience. Here, we tested whether (1) a truth effect is obtained when repetition is only symbolic (i.e., instructed) and whether (2) the experience of factual repetition adds to this symbolic effect.

In two experiments, we examined for the first time how repetition knowledge influences truth judgments in comparison to repetition experience. In Experiment 1, we used a standard truthby-repetition paradigm (i.e., a repetition experience condition), and we compared it with a condition that only instructed participants about repetition without factual prior exposure (i.e., a repetition knowledge condition). Instead, participants saw the statements in the judgment phase only, and the repetition status of these statements depended on whether the statements were allegedly present or absent in an exposure phase underwent by other participants. In this instructed repetition condition, the processing experience should suggest that all the statements are novel, whereas the symbolic information suggests that some are not, and repetition knowledge is under experimental control. Any effect of the symbolic repetition on truth judgments can thus be attributed to non-experiential 
processes, as other potential statement effects of fluency and familiarity should be randomly distributed. Experiment 2 was designed to de-confound the absence of experience with the presence of a repetition tag in the instructed repetition vs. experienced repetition condition. To this aim, we added a third between-participants condition where, after a standard exposure phase, we presented statements in the judgment phase with tags that informed them about the statements' repetition status, similar to the instructed repetition condition.

Both studies also investigated the impact of recognition; that is, the ability of the individual to correctly recognize whether a statement was either repeated or new (i.e., due to factual or instructed repetition). Bacon's (1979) experiments suggest that the individuals' perception of a statement's repetition matters more than its actual repetition status when judging its truth. This finding is consistent with the view that the effect of repetition goes beyond experience, and it emphasizes the importance of memory in the production of the effect. When repetition is only instructed (e.g., "Other participants saw the statement X, which was present [absent] in a prior exposure phase"), the encoding of a statement as either repeated or new has nothing to do with mnemonic traces of the statement itself, nor does its ultimate truth. If recognition moderates the effect of instructed repetition on truth, it does so via deliberate reasoning about the link between repetition and truth. Thus, testing whether recognition can qualify the effect when repetition is either experienced or instructed can provide essential insights on the symbolic component of the effect of repetition on truth.

The pre-registration protocol, materials, experiment scripts, data and analysis code for the two experiments are available on Open Science Framework (Experiment 1: https://osf.io/ynk3s/?view_only=e54c751df507486bb0b6bcdd93508429; Experiment 2: https://osf.io/gacb3/?view_only=2414bf5b320e4c80aaf5d80f1d59bb1d). ${ }^{2}$ No other experiments on

\footnotetext{
2 The anonymized links for the time-stamped pregistrations are available here at https://osf.io/kz56n/?view_only=86d79c511e8d4072a009c897fcb18b62 (Experiment 1) and https://osf.io/fe7xg/?view_only=771e3d94e7044438b5651180ccf8a385 (Experiment 2)
} 
this research question have been conducted so far. We reported all manipulations and measures used in both experiments. Both the studies received formal approval by the local ethics committee.

\section{Experiment 1}

As described, we created two repetition conditions. One randomly assigned group of participants completed a standard, experienced repetition, truth-by-repetition paradigm (see Unkelbach et al., 2019). Participants read a list of statements in an exposure phase and evaluated these repeated and new statements in a later judgment phase. Another group of participants completed the instructed repetition condition. Participants assigned to this condition did not undergo any prior statement exposure phase. Instead, they were instructed that some of the statements they were about to evaluate for truth had been previously presented vs. not presented to other participants. Their task was to evaluate these statements based on how they would have responded if they were exposed to some of those statements in the previous phase. For both repeated and novel statements, we assessed truth judgments and response times. In addition, for each statement, we measured participants' ability to identify whether that statement was either present or absent in the presentation phase (experienced repetition) or said to be present or absent via instructions (instructed condition), and we tested the impact of this variable in both experienced and instructed repetition conditions.

We expected a main effect of repetition on truth. Repeated statements should be judged as more true than new statements overall. In line with prior research (e.g., Unkelbach \& Stahl, 2009; Corneille et al., 2020), the effect should not be qualified by the factual truth of the statement. The critical questions were whether a truth effect would be observed in the instructed repetition condition and whether experienced repetition would increase judgments of truth to a larger extent for participants assigned to the experiential condition. The latter effect is tested with the statement's repetition by repetition condition interaction.

\section{Method}

\section{Design}


We used a repetition condition (instructed vs. factual) $\mathrm{x}$ statement repetition (repeated vs. new) $\mathrm{x}$ factual truth (true vs. false) mixed design, with the first factor manipulated between participants. Truth ratings were the outcome variable.

\section{Sample size determination}

Based on an a priori power analysis, collecting 270 participants allowed detecting an effect size as small as Cohen's $f^{2}=0.039$, which by convenience qualifies as "small" effect size, in a $2 \times 2$ mixed ANOVA, at $\alpha=.05$, and power $1-\beta=.90$. We adopted a sequential analysis approach (Lakens, 2014) with a single interim analysis. Thus, using the Pocock boundary to set the $\alpha$ level, we stopped data collection when 135 participants were collected and analyzed the data. Deciding whether to stop the data collection or double the sample was conditional on the $p$-value $(p<.0294)$ of the test that requires the highest statistical power (i.e., interaction between the effect of statement repetition and repetition condition; see Simonsohn, 2014), as the 3-way interaction was of no theoretical interest.

\section{Materials}

We recruited participants via Prolific Academic and paid 1.00 British Pound for their participation. The experiment was programmed in Inquisit 6. We used 40 statements from Corneille et al. (2020). Half of these statements were factually true, and half were factually false. The statements are available from the online materials.

\section{Procedure}

One hundred and thirty-five participants ( 68 females, $M_{\text {age }}=33.89, S D_{\text {age }}=5.15$ ) completed the study. After entering demographic information, the program assigned participants randomly to the instructed or the experienced repetition condition.

Participants assigned to the experienced condition underwent an exposure phase in which a series of 20 statements appeared individually on screen, written in white on a black background. The 20 statements were randomly selected for each participant from a set of 20 factually true statements and 20 factually false statements. Statements were sequentially presented onscreen for 
$2500 \mathrm{~ms}$ each, with a $1 \mathrm{~s}$ break between statements. Next, participants were introduced to the judgment phase. Participants saw a list of 40 statements (half true and half false), knowing that these statements included both the statements presented in the previous phase and novel statements. Participants' task was to evaluate the extent to which each statement was true/false on a scale ranging from 1 ("completely false") to 6 ("completely true").

In the instructed condition, we informed participants about the study through a cover story in which they received a series of information about a bogus experiment performed by another group of participants. First, the exposure phase of this bogus experiment was described so that participants acquired knowledge about any relevant feature. These features were tightly matched with the features of the exposure operationalized in the experience condition (i.e., number of statements presented, background and text colours, and the timing of exposure). Next came the judgment phase, similar to the one performed by participants in the experienced condition. Crucially, participants knew that this evaluation phase mirrored that of the bogus experiment. Their job was to evaluate the extent to which each statement was true/false, based on how they would have responded if they were exposed to some of those statements in the previous phase. To help them discriminate between previously exposed and novel statements, a sentence saying "This statement was present[absent] in the presentation phase" appeared on the top of the screen upon the occurrence of each statement.

After evaluating the 40 statements, participants in both conditions indicated whether the statement was either absent or present in the presentation phase based on their recollection (experienced condition) or instructions (instructed condition). Finally, participants were thanked for their participation.

\section{Results}


We analysed the data with a generalized mixed model using the 'Imer' function from the 'Ime4 packages from R (Bates et al., 2007). ${ }^{3}$ Participants' truth ratings were the main dependent variable. We tested whether truth judgments varied based on statements' repetition (i.e., old vs. new) factual truth (i.e., factually true vs. false), repetition condition (i.e., experienced vs. instructed) and their interactions (see Figure 1). ${ }^{4}$ Participants judged repeated statements more true $(M=3.98$; $S D=1.46)$ than novel statements $(M=3.59 ; S D=1.45), b=.39, t(5259)=10.20, p<.001$. No significant main effect of factual truth and repetitions condition was found, $b=-.04, t(5259)=-$ $1.08, p=.281$ and $b=-.02, t(132.99)=-.26, p=.792$, respectively. The interaction between statements' repetition and repetition condition was significant, $b=-.61, t(5259)=-7.94, p<.001$. Decomposing this interaction revealed that the effect of statements' repetition was clear in the experienced condition, $t(1299)=12.35, p<0.001, d=.49, \mathrm{BF}_{10}>10^{5}$, but was unconclusive in the instructed condition, $t(1399)=1.76, p=0.08, d=.05, \mathrm{BF}_{10}=.56$. No other significant interaction was found $(p s>.544)$.

Next, we performed the same analyses by including statements' recognition as an additional factor. This variable was derived from comparing statements' repetition and the subjective response at the retention test. Thus, both hits and correct rejections were coded as "correct recognition", whereas false alarms and misses were coded as "incorrect recognition". The percentage of correct recognition was high and comparable across the two conditions (experienced: $89 \%$ vs. instructed: $90 \%$. The analysis yielded a significant main effect of statements' repetition, $b=.39, t(5258.36)=$ $10.22, p<.001$, whereas no other significant main effect was found $(p s>.297)$. There was a significant two-way interaction between statements' repetition and repetition condition, $b=-.61$,

\footnotetext{
${ }^{3}$ We originally planned to conduct our analyses using the 'aov' function in R. However, a generalized mixed model has the advantage to account for intraindividual variance and to allow for the inclusion of a random intercept. Due to the way that variance is partitioned in mixed models, there is no agreement upon a way to calculate standard effect sizes for individual model terms such as main effects or interactions. We therefore decided to report the effect sizes in the form of $b$ estimates.

${ }^{4}$ Although originally pre-registered, we decided not to analyse the impact of our manipulations on reaction times (RTs). In fact, due to how the instructed condition was operationalized (i.e., labels presented on screen along with the statements), participants needed more time to process the whole information.
} 
$t(5258.17)=-8.16, p<.001$, and between statements' repetition and recognition, $b=1.44$, $t(5370.21)=10.93, p<.001$. No other significant two-way interaction emerged $(p \mathrm{~s}>.223)$.

Importantly, we found a significant three-way interaction between statements' repetition, recognition and repetition condition, $b=-.97, t(5370.93)=-3.66, p<.001$. When repetition was correctly recognized, the interaction between statements' repetition and repetition condition was significant, $b=-.72, t(4743.44)=-8.98, p<.001$. Decomposing this interaction showed a significant effect of statements' repetition in both the experienced and the instructed conditions, $t(2307.2)=15.18, p<0.001, d=.63, \mathrm{BF}_{10}>10^{5}$ and $t(2511.4)=3.11, p=0.002, d=.13, \mathrm{BF}_{10}>$ 10, respectively (see Figure 2). Thus, knowing that a statement was repeated increased its judged truth.

When recognition was incorrect, the main effect of statements' repetition was reversed and significant, $b=-.94, t(540.88)=-7.91, p<.001$. This emerged in both the experienced and the instructed condition, $t(217.22)=-6.43, p<0.001, d=.80, \mathrm{BF}_{10}>10^{5}$ and $t(201.35)=-4.34, p<$ $0.001, d=.55, \mathrm{BF}_{10}>10^{2}$ (no significant interaction between statements' repetition and repetition condition, $b=.21, t(540.65)=.90, p=.371)($ see Figure 3$)$. No other significant interaction was found $(p s>.445)$.

\section{Discussion}

In Experiment 1, the experienced repetition condition replicated the standard truth effect: participants judged repeated statements more true than novel statements. The effect of repetition in the instructed repetition condition was not significant overall. Thus, these findings suggest that a substantial component of the truth effect is experiential. However, they also offer initial evidence for a non-experiential component, which is most likely due to deliberate reasoning (i.e., "If a statement is presented twice, then it is probably true"). The moderating role of statements' recognition provides support to this idea. First, the non-experiential contributor was observed when participants encoded the instructed repetition status correctly. Second, a reversal of effects emerged in both the instructed and experienced repetition conditions when participants' recollection of the 
statements' status was incorrect. Whereas in the experienced condition, the effect of recognition conceptually replicates evidence that the perception of statements repetition adds to the truth effect (Bacon, 1979), the impact of recognition in the instructed condition is more informative. Because recognition in the instructed condition is independent of participants' memory for the statements but requires participants' correct (vs. incorrect) encoding and understanding of the instructions, the effect must follow from the symbolic meaning attributed to repetition.

Experiment 1, however, introduced a potential confound. In the judgment phase, a repetition tag was attached to statements in the instruction condition only. As a result, we cannot conclude whether the differential effect observed between experienced and instructed repetition came from the repetition format or from the repetition tag added only in one version (instructed) of the judgment task.

\section{Experiment 2}

Experiment 2 tested (i) the robustness of the instruction-based effect observed in Experiment 1 and (ii) addressed the stated confound in Experiment 1, namely that repetition tags were attached only present in the instructed condition. To do so, we added a third between-subjects condition in our design. This condition was identical to the instructed repetition condition operationalized in Experiment 1, except for the fact that, in the judgment phase, statements appeared on screen along with the tag used to signal statements' repetition status in the instructed condition (i.e., present vs. absent in the presentation phase). In line with Experiment 1, we expected the effect to be stronger when participants made direct experience with statements than when only verbally informed about statements' repetition status. Moreover, we wanted to test the impact of adding an instruction tag to experienced repetition.

On the one hand, the inclusion of the tag could have no impact on the effect of experienced repetition on truth judgments. Bacon (1979, Experiment 2) found evidence of an equivalent increment in credibility when subjects were either informed or uninformed by the experimenter about statements' repetition status. On the other hand, the tag might qualify the effect in two 
opposite ways. First, it might boost the truth effect by enhancing one's knowledge about statements' repetition status. Second, it could reduce the impact of experience on truth judgments by disrupting a spontaneous, holistic processing of participants' experience of fluency (Whittlesea \& Price, 2001). Finally, adding this condition addresses the moderating role of recognition found in Experiment 1. As pointed out, the processes responsible for answering the memory question might have differed between the two repetition conditions used in Experiment 1. In the experienced condition, several factors contribute to a "present" response (e.g., feelings of familiarity or fluency, as well as conscious recollection). Instead, participants in the instructed condition could respond "present" without any specific recollection of the statement. Adding tags to statements in the judgment phase of the experienced condition allows a ceteris paribus test of the recognition influence on truth judgments when repetition is either merely experienced or also instructed.

\section{Method}

\section{Design}

We used a repetition condition (instructed vs. experienced-only vs. experienced plus tag) $\mathrm{x}$ repetition (repeated vs. new) $\mathrm{x}$ factual truth (true vs. false) mixed design, with the first factor manipulated between subjects. Truth judgments were the dependent variable.

\section{Sample size determination}

We followed the same sequential analysis approach (Lakens, 2014) used in Experiment 1. We powered the study on the difference in the truth effect (i.e., the average difference of "repeated" minus "new" statements, collapsed across factual truth) observed in two critical contrasts. The first contrast concerned the comparison between the instructed condition $(-2)$ with the average effect observed in the experienced-only $(+1)$ and experienced plus tag $(+1)$ conditions. The second contrast tested whether the difference in the truth effect between experienced-only and experienced plus tag. We aimed at a sample size that could detect effects as small as Cohen's $f^{2}=0.039$. We used the "pwr" package in R to estimate the number of participants required in each cell, at $\alpha=.05$, and power $1-\beta=.90$ (i.e., to obtain an actual power $=.90$, the nominal power of each contrast was 
adjusted to .95). The analysis suggested 111 participants per group, leading to 333 participants. We planned a single interim analysis and stopped data collection when 167 participants were recruited. Because one of the two contrasts did not reach the set significance level $(p<.0294)$, we collected the remaining 166 participants.

\section{Materials}

Participants were recruited via Prolific Academic and paid 1.00 British Pound for their participation. The experiment was programmed in Inquisit 6 . The statements were identical to those used in Experiment 1.

\section{Procedure}

Three hundred and thirty-three participants (188 females, $\left.M_{\text {age }}=34.88, S D_{\text {age }}=5.04\right)$ completed the study. The experimental procedure replicated Experiment 1, except for the inclusion of the experienced repetition plus tag condition. Participants assigned to this condition underwent the same exposure and judgment phase administered in the experienced-only condition (see Experiment 1). However, the 40 statements presented in the judgment phase were presented onscreen with the same tag used in the instruction condition to signal whether the statement was either absent or present in the prior exposure phase.

\section{Results}

Like for Experiment 1, we analyzed the data with a generalized mixed model, using the 'Imer' function in R. Truth judgment was the main dependent variable. We set the two critical contrast and tested and included them in the model as predictors with statements' repetition. As Experiment 1 indicated no effect of statements' factual truth, we planned not to include this variable in the analysis. ${ }^{5}$. Participants judged repeated statements more true $(M=4.13 ; S D=1.46)$ than novel statements $(M=3.61 ; S D=1.47), b=.52, t(12984)=21.33, p<.001$ (see Figure 4). The main effect of repetition condition in the first contrast was significant, $b=.21, t(330)=4.06, p<$

\footnotetext{
${ }^{5}$ Adding this variable to the analyses had no impact on the results.
} 
.001 , and so was in the second contrast, $b=.12, t(330)=1.96, p=.050$. Regardless of whether they were repeated or new, statements in the two experienced conditions were judged (on average) more true relative to the same statements presented in the instructed condition (first contrast). Moreover, participants rated truth higher for statements in the experienced plus tag condition than in the experienced-only condition (second contrast)

More central to our purpose, we found a significant interaction between the first contrast and statements' repetition, $b=.21, t(12984)=4.12, p<.001$. This interaction revealed that the effect in the two collapsed experienced conditions was stronger than that observed in the instructed condition, although the effect of repetition was significant in both cases, $t(4479)=19.77, p<0.001$, $d=.41, \mathrm{BF}_{10}>10^{5}$ and $t(2179)=8.45, p<0.001, d=.26, \mathrm{BF}_{10}>10^{5}$, respectively. Thus, we confirmed that a prominent component of the effect of repetition on truth is experiential, but we also found evidence for an the hypothesized symbolic component. The second contrast was not moderated by repetition, $b=.10, t(12984)=1.64, p=.101$, indicating a comparable effect in the experienced-only and in the experienced plus tag conditions; $t(2259)=12.84, p<0.001, d=.38$, $\mathrm{BF}_{10}>10^{5}$ and $t(2219)=15.16, p<0.001, d=.43, \mathrm{BF}_{10}>10^{5}$, respectively.

Next, we performed the same analyses by including statements' recognition as an additional factor. The percentage of correct recognition was high and comparable across the three conditions (experienced-only: 89\%; experienced plus tag: 93\%; instructed: 94\%). The analysis showed significant main effects of both repetition and recognition, $b=.52, t(12982.59)=21.27, p<.001$, and $b=.17, t(13081.11)=3.32, p<.001$, respectively. The main effect of repetition in the first contrast was significant, $b=.23, t(331.12)=4.49, p<.001$, but it was not in the second contrast, $b$ $=.11, t(330.27)=1.93, p=.055$. The interaction between the first contrast term (i.e., averaged experienced-only and experienced plus tag vs. instructed condition) and repetition was significant, $b$ $=.25, t(12983.25)=4.80, p<.001$. The interaction between the second contrast (i.e., experiencedonly vs. experienced plus tag) and repetition was not significant, $b=.06, t(12981.91)=1.05, p=$ 
Consistent with Experiment 1, we also found an interaction between repetition and recognition, $b=1.15, t(13306.27)=11.41, p<.001$. In all three conditions, the effect of repetition on judgments of truth was reversed when repetition was incorrectly recognized (instructed: $t(217.14)=-4.04, p<.001, d=.54 ;$ experienced-only: $t(411.53)=-2.94, p=.003, d=.28 ;$ experienced plus tag: $t(292.5)=-4.55, p<.001, d=.53$ ) (see Figure 5 and Figure 6 for the effect on correct and incorrect trials, respectively). Thus, when tags were added to statements in the experienced condition, incorrect recognition led to an effect that was comparable to that observed in the instructed condition. Also significant was the interaction between the second contrast and recognition, $b=-.33, t(12955.93)=-2.79, p=.005$. No significant interaction was found between the first contrast and recognition, $b=-.09, t(13158.37)=-.76, p=.447$.

A significant three-way interaction involving the second contrast, repetition and recognition was found, $b=.52, t(13306.39)=2.27, p=.023$. Decomposing this interaction indicated that, when participants remembered correctly whether a statement was present or absent, a numerical difference in the relevant direction emerged, although it was not significant, $b=.11, t(12099)=$ $1.72, p=.085$. No interaction between repetition and the second contrast was found for incorrect trials, $b=-.27, t(954.62)=-1.24, p=.216$. The three-way interaction involving the first contrast, repetition and recognition was not significant, $b=.14, t(13306.17)=.61, p=.540$.

\section{Discussion}

Experiment 2 confirms that mere knowledge about repetition without factual repetition contributes to the TBR effect. This is indicated by a truth effect in the instructed condition and a reversal of effects when repetition is incorrectly recognized. However, the current findings also support the unique contribution of experiential processes to the truth effect. The effect was larger in the experienced-repetition than in the instructed-repetition condition. In addition, this difference cannot be attributed to the confound between the absence of experience and the presence of a tag that was present in Experiment 1.

\section{Combined results (not pre-registered)}


As the overall effect of repetition in the instructed condition was conclusive and significant in Experiment 2 but inconclusive in Experiment 1, we conducted a meta-analysis of the two studies. ${ }^{6}$ As both studies were pre-registered and these are the only two studies in this line of research so far, the internal meta-analysis is justified (see Vosgerau et al., 2019). We combined the data from the two experiments, including only the instructed and the experienced-only conditions, but not the experience plus tag condition introduced in Experiment 2. This led to a total sample of 357 participants (179 in the instructed condition and 178 in the experienced condition). We analyzed the data with a generalized mixed model. We entered repetition (i.e., old vs. new), repetition condition (i.e., experienced vs. instructed), and their interaction as predictors in the model. We found a main effect of statements' repetition, $b=.43, t(13921)=18.43, p<.001$, with participants judging repeated statements more true $(M=4.01 ; S D=1.45)$ than novel statements $(M$ $=3.58 ; S D=1.46)$. There was a significant effect of repetition condition, $b=-.10, t(355)=-2.31, p$ $=.022$. The interaction between statements' repetition and repetition condition was significant, $b=$ $.33, t(13921)=-7.08, p<.001$. Decomposing this interaction revealed that, although larger in the experienced condition, the effect of statements' repetition was significant in both the experienced and in the instructed conditions, $t(3559)=17.71, p<0.001, d=.42, \mathrm{BF}_{10}>10^{5}$ and $t(3579)=7.70$, $p<0.001, d=.18, \mathrm{BF}_{10}>10^{5}$, respectively. Thus, the two studies overall offer convincing evidence for a truth effect without actual repetition and a stronger effect of factual compared to instructed repetition on judged truth. This conclusion is also supported in both studies separately when considering correct memory responses only (the incorrect recollection responses leading to a reversal of the truth effect).

\section{General discussion}

\footnotetext{
${ }^{6}$ Upon further consideration we realized that, whereas our power analyses in both the studies were well planned to detect the interaction effect(s) between repetition condition and statements' repetition, they were not for the simple effect of repetition in the instructed condition. In fact, in Experiment 1, following our pre-registered plan to stop the data collection based on the interaction term left us with an overall sample that was too small to observe a significant instructed effect.
} 
Deciding whether information is true or false is a challenging task. Extensive research showed that one key variable that people often use to judge the truth of a statement is repetition (e.g., Hasher et al. 1977): repeated statements are judged more true than new ones (see Dechêne et al., 2010). Virtually all explanations of this truth effect refer to the processing consequences of repetition, that is, higher recognition rates compared to new statements, higher familiarity, and higher fluency (see Unkelbach et al., 2019). However, in many natural communication situations, people get to know that a statement is repeated (e.g., it occurred frequently) without prior exposure to the statement. Here, we asked whether repetition can be used as a cue for truth without prior exposure, and thus, in the absence of experiential consequences of repetition such as fluency.

Two experiments tested the impact of experienced vs. instructed statements' repetition on judgments of truth. Experiment 1 found that the kind of repetition moderated the effect of repetition on truth. Participants in the experienced repetition condition showed a significantly stronger truth effect than those for whom repetition was just instructed. However, we also found evidence for an effect of repetition on truth judgments in the instructed condition. Although only descriptive when considered across all the trials, this instruction-based effect was significant when considering only the (more diagnostic) statements correctly recognized as either repeated or new in a retention test. Experiment 2 replicated the main findings of Experiment 1 and tested the potential impact of the presence of an instructional tag in the experienced repetition condition. We found that, overall, repetition had a stronger effect on truth when experienced than when instructed. However, the instructed repetition effect was significant, therefore confirming a non-experiential contribution to the effect.

One key variable determining whether repeated statements are judged to be true or false is the resulting experience when processing those statements. However, when this experiential component and repetition were dissociated, participants still relied on repetition to infer statements' truth value. This reliance on instructed repetition to infer truth may have several sources. For example, people might learn an ecological correlation between repetition and truth (Unkelbach, 
2006, 2007). Thus, because truth and repetition are correlated, people judge statements that occur more frequently to have a higher probability of being true, and vice-versa (for similar arguments, see Unkelback \& Greifeneder, 2013, and Reber \& Unkelbach, 2010; for a reversal of the truth effect in alternative ecologies, see Corneille et al., 2020). Similarly, people might have lay theories about their processing experiences (e.g., if it is easy to process, it is likely true) and judge the statements accordingly (e.g., Schwarz, 2004). In Experiment 2, we found that instructions did not add significantly to the effect of experience on truth judgments. However, it is likely that remembering whether a statement was repeated or new was relatively easy. Under such conditions, instructions add little to the subjective experience of recognizing a statement. However, the direct comparison between the two experienced conditions on trials correctly recognized indicated a descriptively stronger effect in the experienced plus tag condition, although this comparison did not reach conventional significance levels. Future investigations should test how instructions could complement experience when recognizing whether a statement is repeated or not is more complex (e.g., the time delay between exposure and judgment phase).

Another important finding was the reversal effect when participants were incorrect in indicating statements' repetition status. This reversal happened independent of the between-subjects conditions operationalized across the two experiments. In the experienced repetition, the role played by statements' recognition is in line with prior evidence (Bacon, 1979). Unkelbach and Stahl (2009) also isolated a specific decrease in subjective truth for new statements rather than a sole increase for old statements. However, this finding does not oppose a fluency explanation. Based on the recent referential theory proposed by Unkelbach and Rom (2017), people judge truth based on coherent references for statements in memory. When the elements of a statement have no references, the prior presentation may instigate and link new references. Because of this, repeated statements have a higher chance of being judged as true. Notably, the model implies that factually new (old) statements can be judged as true (false) if coherent references in memory can (cannot) be found. With that being said, showing that both the experienced and the instructed effects are qualified by 
the same variable suggests that the two share a common deliberate component that acts upon the link between repetition and truth (e.g., "if a statement is repeated, then it must be true"), which might be explicitly available (i.e., a naïve theory; Schwarz, 2004) or learned without conscious access (i.e., a learned correlation; Unkelbach, 2006). Along this line, one could argue that because repetition and truth correlate, the effect of one variable on the other is potentially bidirectional. If this is the case, then recognition could also be influenced by the level of truth assigned to each statement (e.g., "if I judged it true, then it must be repeated"; see Nahon et al., 2021).

Our findings suggest that the proximal cues responsible for the effect of experienced vs. instructed repetition might differ. Namely, in the experienced repetition, truth judgments should be conditional upon the positive correlations between (i) fluency and truth and (ii) repetition and truth. Instead, in the case of instructed repetition, only the latter correlation should qualify the effect. Nevertheless, some might ask whether the effect of instructed repetition can be entirely dissociated from fluency. Namely, because repetition and fluency are strongly associated, one could hypothesize that instructed repetition could also affect truth via simulated fluency (Mrkva et al., 2017; Risen \& Critcher, 2011). To answer this question, one would need to manipulate the link between fluency and truth. Prior research has shown that when the correlation between the experience of fluency and truth is manipulated to be negative, a reversal in the truth effect is observed (e.g., Unkelbach, 2007; see also Olds \& Westerman, 2012). Testing the differential impact of this variable on both experienced and instructed repetition could provide additional insights into the processes through which instructed repetition affects judgments of truth. If instruction-based and experience-based truth effects depend on the validity of distinct cues, then manipulating the validity of the fluency cue should leave the effect of instructed repetition on truth unaffected.

Another relevant issue concerns the impact of subjective knowledge about the factual truth of a statement and its relationship with the effect of repetition. Consistently with prior research (e.g., Corneille, 2020; Fazio et al., 2019), we found that whether statements were judged to be true or false did not depend on their factual truth. Although this null effect might be due to participants' 
lack of knowledge, prior studies have shown that the experience of fluency is still used as a valid cue for truth when knowledge about statements' content (Fazio et al., 2015; Fazio et al., 2019), source credibility (Begg et al., 1992; Unkelbach \& Stahl, 2009), and declarative advice about the credibility of a statement (Unkelbach \& Greifeneder, 2018) suggest the opposite. This is also illustrated in a recent study that found a truth effect for highly implausible statements, such as “earth is a perfect square", for which knowledge (of falsity) is present (Lacassagne et al., 2021).

However, those studies did not clarify whether it was the fluency experience induced by statements' repetition (i.e., "It feels fluent, therefore it must be true") or repetition per se (i.e., "It is repeated, therefore it must be true") that explains why repeated statements are believed more than novel statements despite their inconsistency with knowledge. Discerning between factual and instruction-based truth effects can answer this question. If repetition affects truth despite conflicting information due to internal cues made available by the experience of fluency, then only factual repetition, but not instructed repetition, should get the better of such inconsistent information.

Last, the current findings have real-life implications. We found that in the absence of experiential cues, knowing that information has been repeated suffices to convey credibility. This shows that even without direct experience, repetition is a cue that people use to make decisions. As we have argued above, the impact of repetition presented in the form of verbal instructions is likely due to deliberate reasoning. Such deliberate reasoning can be easily represented in the form of a syllogism: "Repeated information is true"; "Statement X is repeated"; "Statement X is true". To become more efficient as decision-makers, people must be aware of the fallacy of those premises. First, the overlap between repetition and truth is far from perfect but simply probabilistic (Unkelbach \& Greifeneder, 2013). For instance, before Pythagoras, many used to claim that the earth was flat, although the statement was as false then as it is now. However, not only this tendency to infer that something is true just because it is repeated persists, but it is also exploited for persuasive motives. This has direct consequences for the validity of the second premise. Even more than experienced-repetition, instructed repetition can be easily manipulated. For instance, to support 
a new anti-immigration law, a political leader might state that people have repeatedly said that "the more the immigrants, the higher the crime rate". If truth judgments are biased by simple pieces of information about the repetition status of a statement, then questioning the link between repetition and truth becomes key for using strategies that leads to more valid cues to determine whether that statement is credible (e.g., fact-checking).

\section{Conclusion}

This work represents the first attempt to assess the impact of instructed repetition on truth judgments. In two experiments, we showed that the truth effect was stronger when repetition was factual rather than merely instructed. However, we provided initial evidence that a component of the effect is unrelated to the repetition experience. A truth effect was still detectable in the absence of any internal cue (i.e., fluency) induced by the factual repetition of the statement. In addition, the truth effect was systematically reversed in both studies and in all conditions when participants' memory was incorrect. We propose that this non-experiential component can be explained in light of the validity of repetition to act as a valid cue for truth and should be therefore conditional upon the learning history. This finding paves the way for new research avenues interested in isolating the unique contribution of known repetition and experienced fluency on truth judgments. 


\section{References}

Arkes, H. R., Hackett, C., \& Boehm, L. (1989). The generality of the relation between familiarity and judged validity. Journal of Behavioral Decision Making, 2(2), 81-94. https://doi.org/10.1002/bdm.3960020203

Bacon, F. T. (1979). Credibility of repeated statements: Memory for trivia. Journal of Experimental Psychology: Human Learning and Memory, 5(3), 241-252. https://doi.org/10.1037/02787393.5.3.241

Bates, D., Sarkar, D., Bates, M. D., \& Matrix, L. (2007). The lme4 package. $R$ package version, 2(1), 74 .

Begg, I. M., Anas, A., \& Farinacci, S. (1992). Dissociation of processes in belief: Source recollection, statement familiarity, and the illusion of truth. Journal of Experimental Psychology: General, 121(4), 446-458. https://doi.org/10.1037/0096-3445.121.4.446

Bornstein, R. F. (1989). Exposure and affect: overview and meta-analysis of research, 19681987. Psychological bulletin, 106(2), 265-289. https://doi.org/10.1037/0033-2909.106.2.265

Clore, G. L. (1992). Cognitive phenomenology: Feelings and the construction of judgment. The construction of social judgments, 10, 133-163.

Corneille, O., Mierop, A., \& Unkelbach, C. (2020). Repetition increases both the perceived truth and fakeness of information: An ecological account. Cognition, 205, 104470. https://doi.org/10.1016/j.cognition.2020.104470

Corneille, O., Mierop, A., Stahl, C., \& Hütter, M. (2019). Evidence suggestive of uncontrollable attitude acquisition replicates in an instructions-based evaluative conditioning paradigm: Implications for associative attitude acquisition. Journal of Experimental Social Psychology, 85, 103841. https://doi.org/10.1016/j.jesp.2019.103841

De Houwer, J., \& Hughes, S. (2016). Evaluative conditioning as a symbolic phenomenon: On the relation between evaluative conditioning, evaluative conditioning via instructions, and persuasion. Social Cognition, 34(5), 480-494. https://doi.org/10.1521/soco.2016.34.5.480 
Dechêne, A., Stahl, C., Hansen, J., \& Wänke, M. (2010). The truth about the truth: A meta-analytic review of the truth effect. Personality and Social Psychology Review, 14(2), 238-257. https://doi.org/10.1177/1088868309352251

DiFonzo, N., Beckstead, J. W., Stupak, N., \& Walders, K. (2016). Validity judgments of rumors heard multiple times: The shape of the truth effect. Social Influence, 11(1), 22-39. https://doi.org/10.1080/15534510.2015.1137224

Fazio, L. K., Brashier, N. M., Payne, B. K., \& Marsh, E. J. (2015). Knowledge does not protect against illusory truth. Journal of Experimental Psychology: General, 144(5), 993-1002. http://dx.doi.org/10.1037/xge0000098

Fazio, L. K., Rand, D. G., \& Pennycook, G. (2019). Repetition increases perceived truth equally for plausible and implausible statements. Psychonomic Bulletin \& Review, 26(5), 1705-1710. https://doi.org/10.3758/s13423-019-01651-4

Hasher, L., Goldstein, D., \& Toppino, T. (1977). Frequency and the conference of referential validity. Journal of Verbal Learning and Verbal Behavior, 16(1), 107-112. https://doi.org/10.1016/S0022-5371(77)80012-1

Johar, G. V., \& Roggeveen, A. L. (2007). Changing false beliefs from repeated advertising: The role of claim-refutation alignment. Journal of Consumer Psychology, 17(2), 118-127. https://doi.org/10.1016/S1057-7408(07)70018-9

Lacassagne, D., Béna, J., \& Corneille, O. (2021). Is Earth a Perfect Square? Repetition Increases the Perceived Truth of Highly Implausible Statements. https://doi.org/10.31234/osf.io/fce8z

Lakens, D. (2014). Performing high-powered studies efficiently with sequential analyses. European Journal of Social Psychology, 44(7), 701-710. https://doi.org/10.1002/ejsp.2023

Montoya, R. M., Horton, R. S., Vevea, J. L., Citkowicz, M., \& Lauber, E. A. (2017). A reexamination of the mere exposure effect: The influence of repeated exposure on recognition, familiarity, and liking. Psychological bulletin, 143(5), 459-498.

https://doi.org/10.1037/bul0000085 
Mrkva, K., Travers, M., \& Van Boven, L. (2018). Simulational fluency reduces feelings of psychological distance. Journal of Experimental Psychology: General, 147(3), 354-376. https://doi.org/10.1037/xge0000408

Nahon, L. S., Teige-Mocigemba, S., Reber, R., \& Greifeneder, R. (2021). Truth feels easy: Knowing information is true enhances experienced processing fluency. Cognition, 215, 104819. https://doi.org/10.1016/j.cognition.2021.104819

Nelson, T. O. (1996). Consciousness and metacognition. American psychologist, 51(2), 102-116. https://doi.org/10.1037/0003-066X.51.2.102

Olds, J. M., \& Westerman, D. L. (2012). Can fluency be interpreted as novelty? Retraining the interpretation of fluency in recognition memory. Journal of Experimental Psychology: Learning, Memory, and Cognition, 38(3), 653-664. https://doi.org/10.1037/a0026784

Orne, M. T. (1962). On the social psychology of the psychological experiment: With particular reference to demand characteristics and their implications. American Psychologist, 17(11), 776-783. https://doi.org/10.1037/h0043424

Reber, R., \& Schwarz, N. (1999). Effects of perceptual fluency on judgments of truth. Consciousness and Cognition, 8(3), 338-342. https://doi.org/10.1006/ccog.1999.0386

Reber, R., \& Unkelbach, C. (2010). The epistemic status of processing fluency as source for judgments of truth. Review of Philosophy and Psychology, 1(4), 563-581. https://doi.org/10.1007/s13164-010-0039-7

Risen, J. L., \& Critcher, C. R. (2011). Visceral fit: While in a visceral state, associated states of the world seem more likely. Journal of personality and social psychology, 100(5), 777-793. https://doi.org/10.1037/a0022460

Simonsohn, U. (2014). No-way interactions. https://doi.org/10.15200/winn.142559.90552.

Unkelbach, C. (2006). The learned interpretation of cognitive fluency. Psychological Science, 17(4), 339-345. https://doi.org/10.1111/j.1467-9280.2006.01708.x 
Unkelbach, C. (2007). Reversing the truth effect: Learning the interpretation of processing fluency in judgments of truth. Journal of Experimental Psychology: Learning, Memory, and Cognition, 33(1), 219-230. https://doi.org/10.1037/0278-7393.33.1.219

Unkelbach, C., \& Greifeneder, R. (2018). Experiential fluency and declarative advice jointly inform judgments of truth. Journal of Experimental Social Psychology, 79, 78-86. https://doi.org/10.1016/j.jesp.2018.06.010

Unkelbach, C., \& Greifeneder, R. (Eds.). (2013). The experience of thinking: How the fluency of mental processes influences cognition and behaviour. Psychology Press.

Unkelbach, C., \& Rom, S. C. (2017). A referential theory of the repetition-induced truth effect. Cognition, 160, 110-126. https://doi.org/10.1016/j.cognition.2016.12.016

Unkelbach, C., \& Stahl, C. (2009). A multinomial modeling approach to dissociate different components of the truth effect. Consciousness and Cognition, 18(1), 22-38. https://doi.org/10.1016/j.concog.2008.09.006

Unkelbach, C., Koch, A., Silva, R. R., \& Garcia-Marques, T. (2019). Truth by repetition: Explanations and implications. Current Directions in Psychological Science, 28(3), 247253. https://doi.org/10.1177/0963721419827854

Van Dessel, P., Mertens, G., Smith, C. T., \& De Houwer, J. (2017). The mere exposure instruction effect. Experimental psychology. 64(5), 299-314. https://doi.org/10.1027/1618$3169 / \mathrm{a} 000376$

Vosgerau, J., Simonsohn, U., Nelson, L. D., \& Simmons, J. P. (2019). 99\% impossible: A valid, or falsifiable, internal meta-analysis. Journal of Experimental Psychology: General, 148(9), 1628-1639. https://doi.org/10.1037/xge0000663

Whittlesea, B. W., \& Price, J. R. (2001). Implicit/explicit memory versus analytic/nonanalytic processing: Rethinking the mere exposure effect. Memory \& Cognition, 29(2), 234-246. https://doi.org/10.3758/BF03194917 
Winkielman, P., Schwarz, N., Fazendeiro, T., \& Reber, R. (2003). The hedonic marking of processing fluency: Implications for evaluative judgment. In J. Musch \& K. C. Klauer (Eds.),The psychology of evaluation. Affective processes in cognition and emotion (pp. 189217). Mahwah, NJ: Lawrence Erlbaum Associates, Inc.

Zajonc, R. B. The attitudinal effects of mere exposure. Journal of Personality and Social Psychology, 1968, 8 (2, Pt. 2). https://doi.org/10.1037/h0025848 

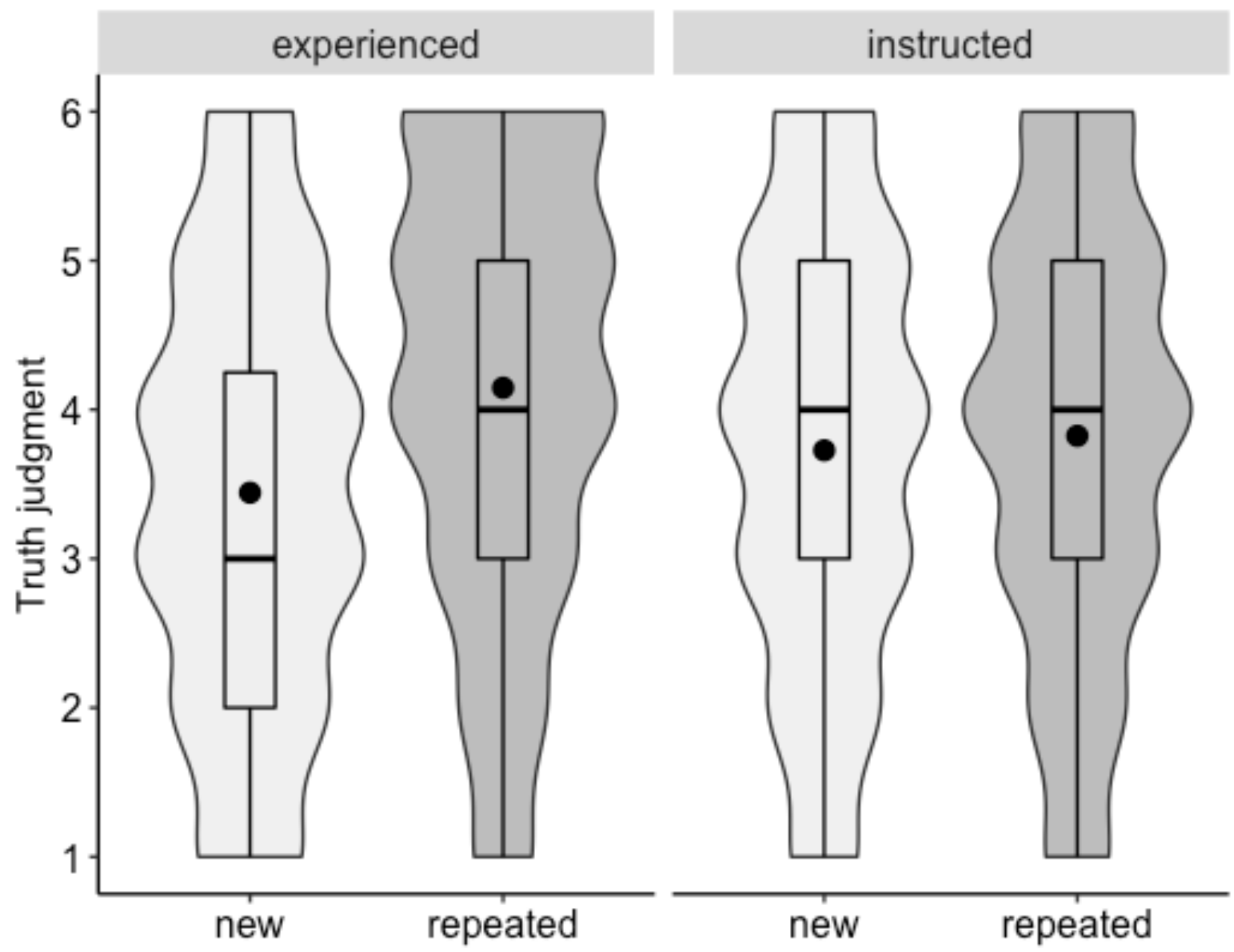

Figure 1. Overall effect of statements' repetition on truth - Experiment 1 (dots represent the mean) 

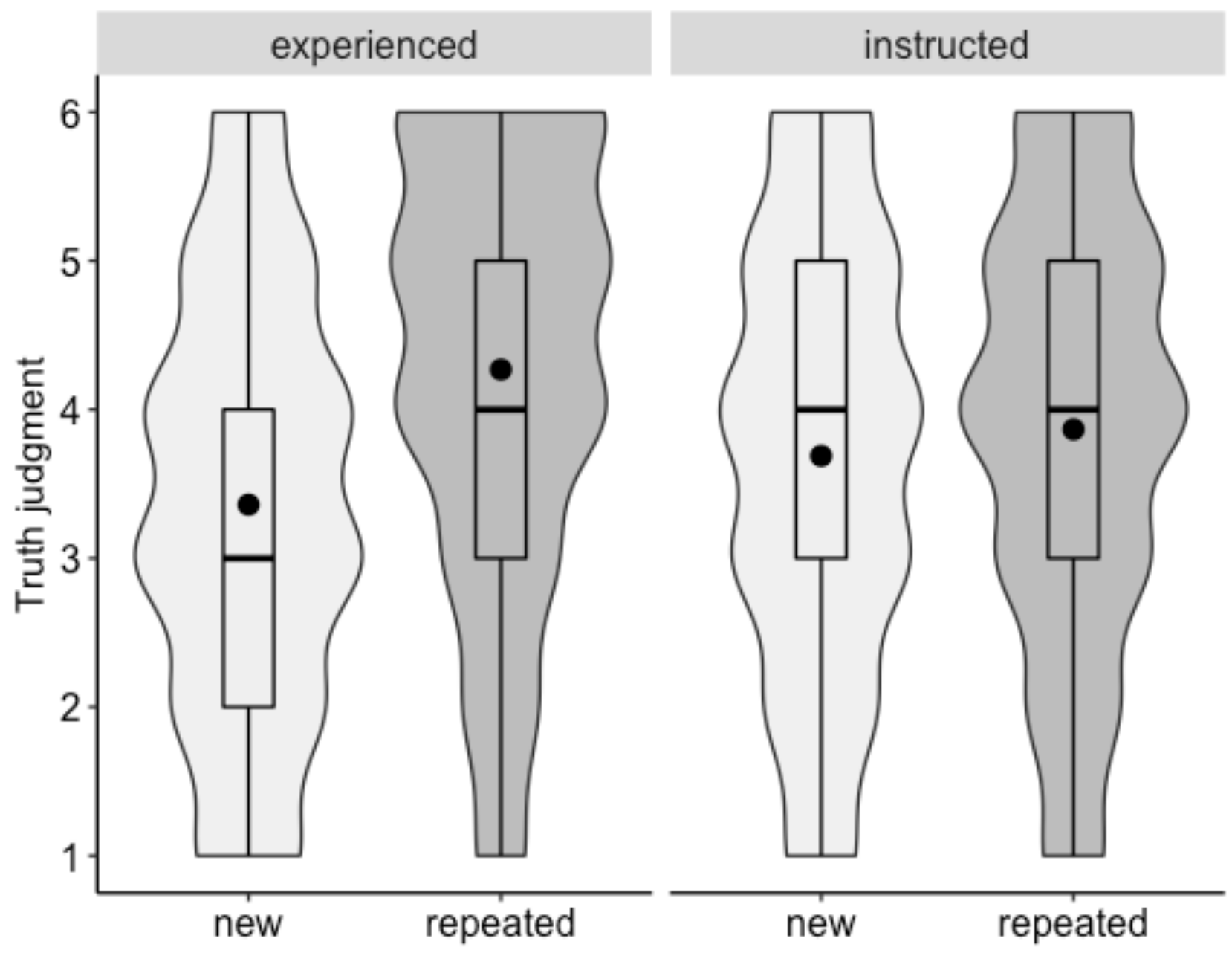

Figure 2. Effect of statements' repetition on truth for correct recognition - Experiment 1 

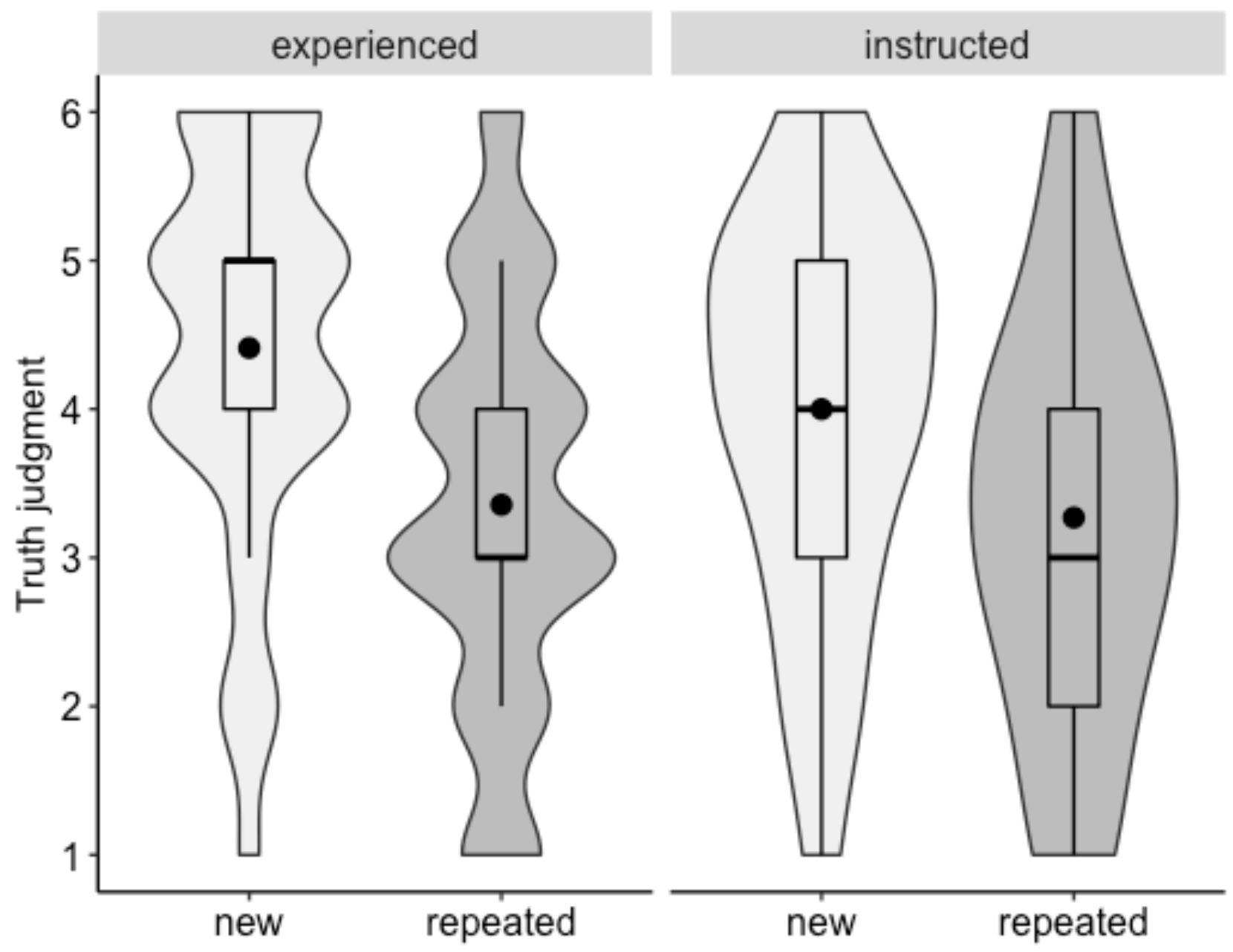

Figure 3. Effect of statements' repetition on truth for incorrect recognition - Experiment 1 

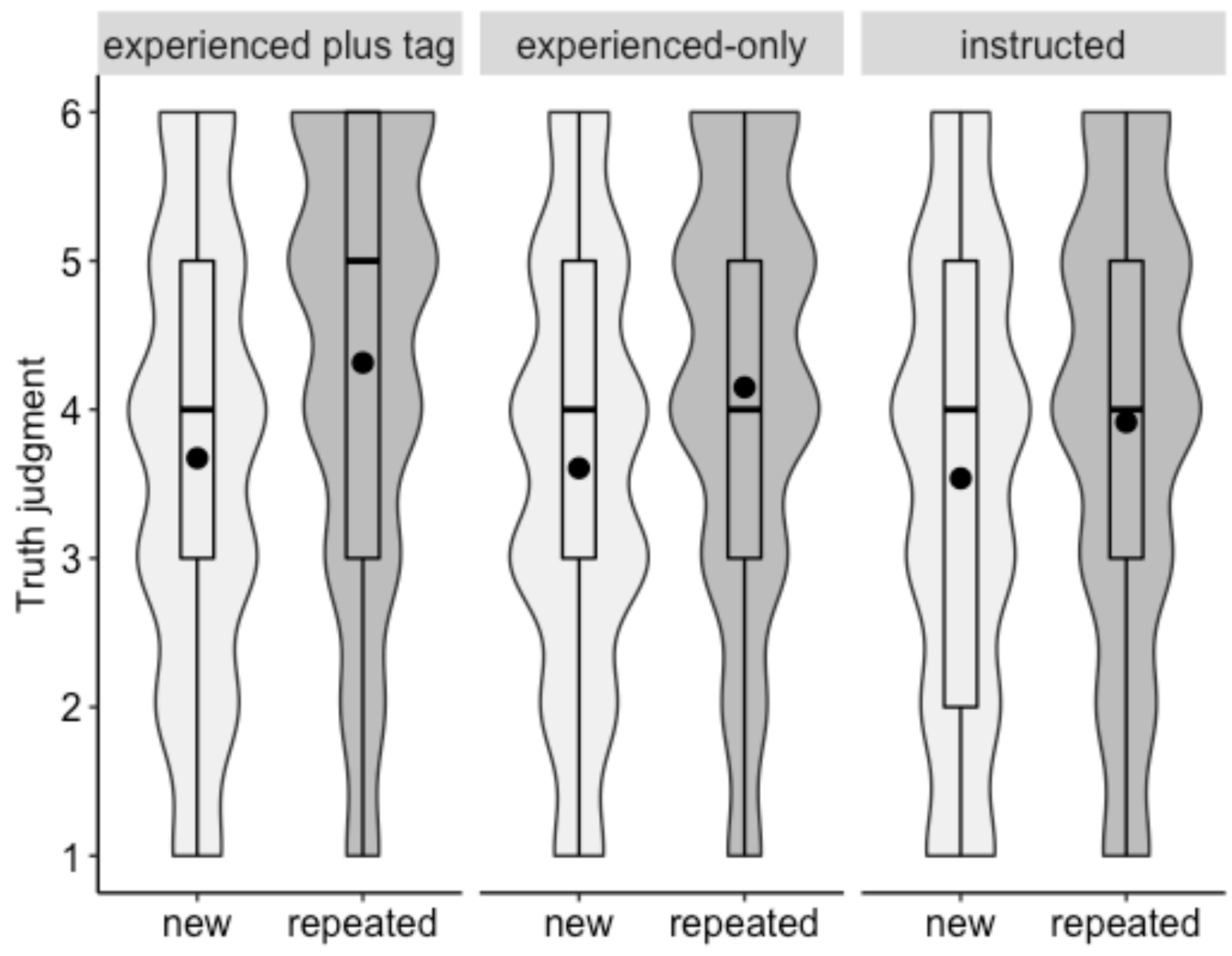

Figure 4. Overall effect of statements' repetition on truth - Experiment 2 


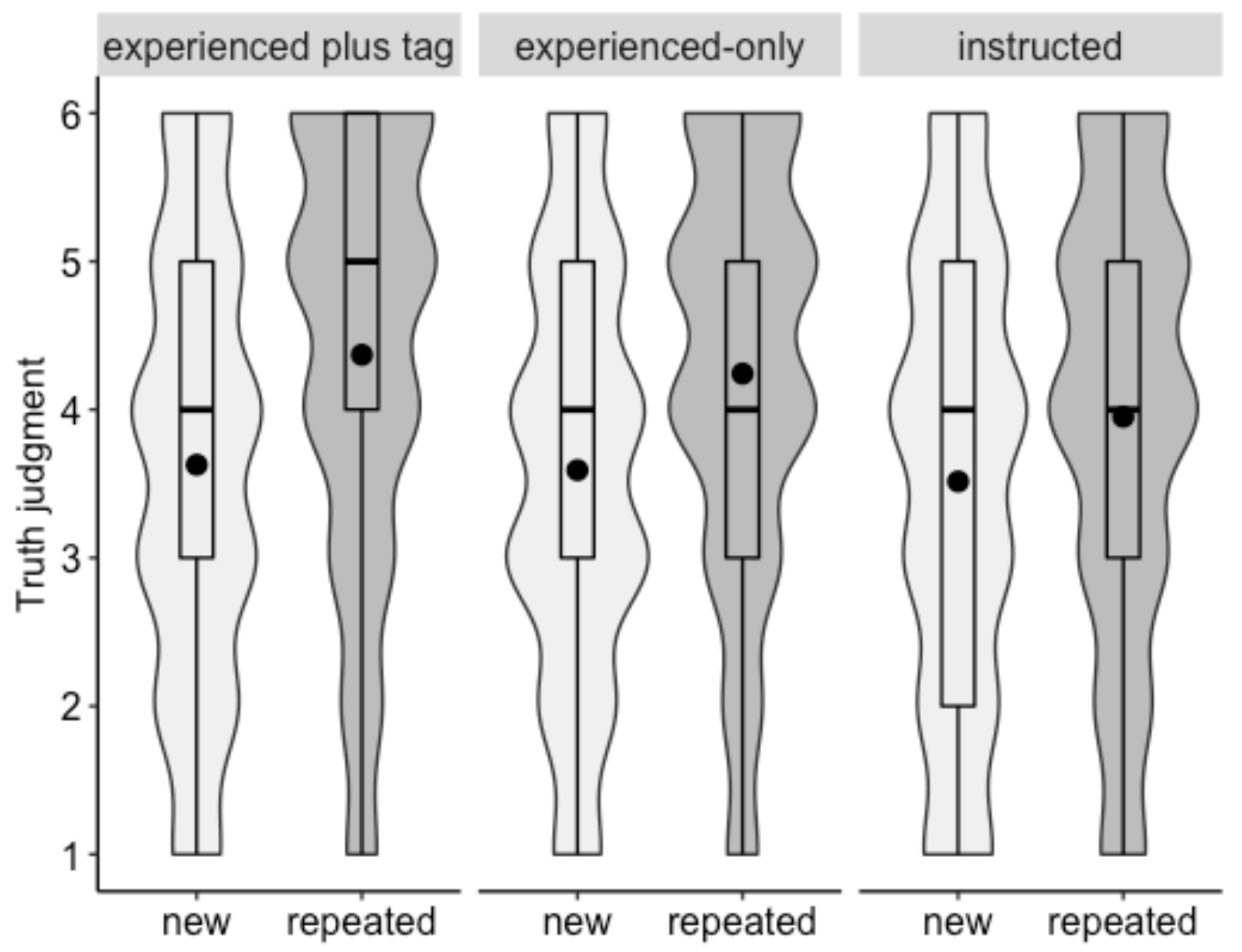

Figure 5. Effect of statements' repetition on truth for correct recognition - Experiment 2 

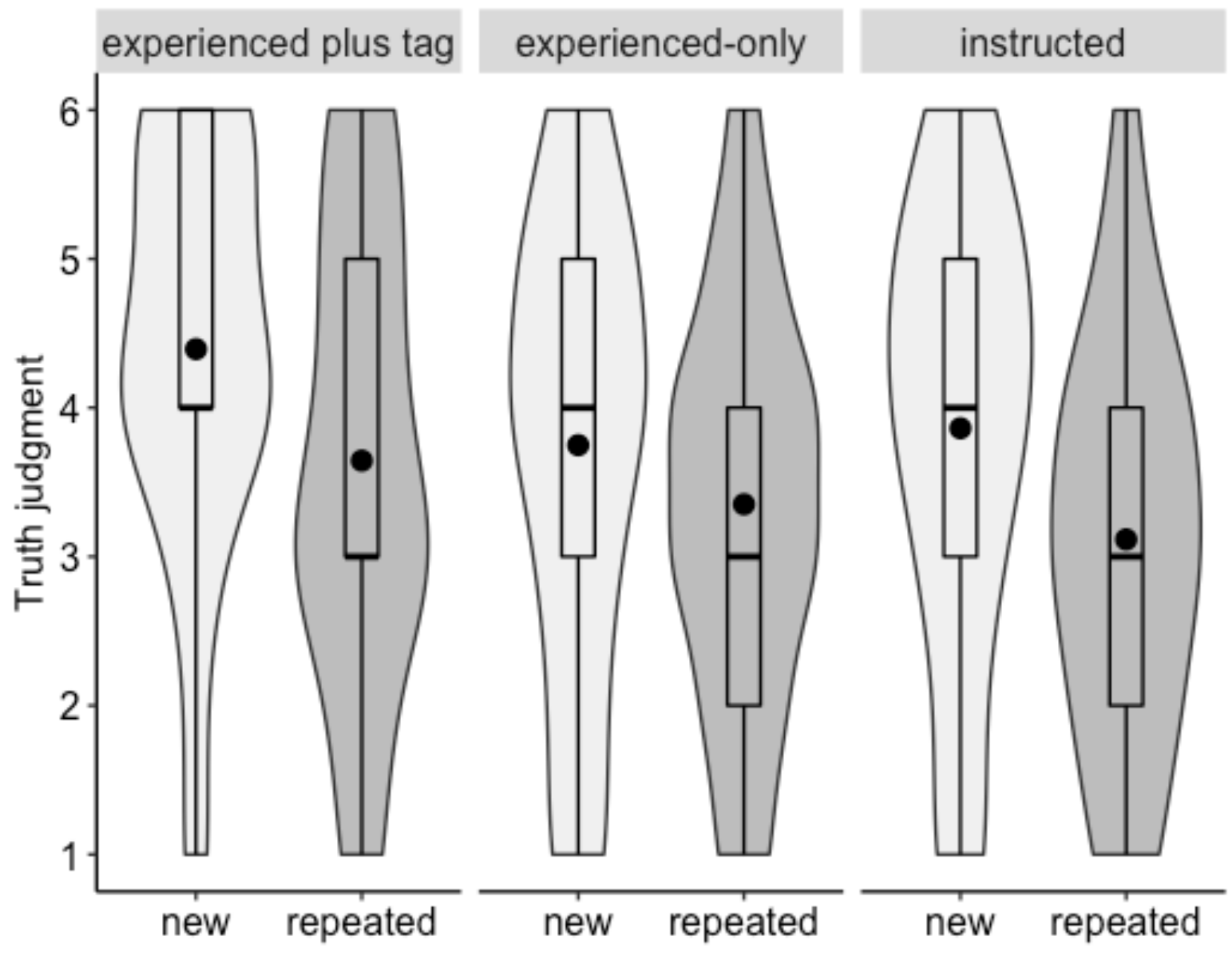

Figure 6. Effect of statements' repetition on truth for incorrect recognition - Experiment 2 